\title{
AFFINE LIE ALGEBRAS AND HECKE MODULAR FORMS
}

\author{
BY V. G. KAC AND D. H. PETERSON
}

The character of a highest weight representation of an affine Lie algebra can be written as a finite sum of products of classical $\Theta$-functions and certain modular functions, called string functions. We find the transformation law for the string functions, which allows us to compute them explicitly in many interesting cases. Finally, we write an explicit formula for the partition function, in the simplest case $A_{1}^{(1)}$, and compute the string functions directly. After multiplication by the cube of the $\eta$-function, they turn out to be Hecke modular forms!

1. (See [3] or [7] for details.) Let $g$ be a complex finite-dimensional simple Lie algebra, $\bar{g}$ a Cartan subalgebra of $g$. $\Delta$ the set of roots of $\bar{g}$ in $g . \Delta_{+}$a set of positive roots, $\Pi=\left\{\alpha_{1}, \ldots, \alpha_{l}\right\}$ the corresponding set of simple roots, $\theta$ the highest root. Let $($,$) be an invariant symmetric bilinear form on g$ normalized by $(\theta, \theta)=2$. For $\alpha \in \mathfrak{G}^{*}$ with $(\alpha, \alpha) \neq 0$ define $H_{\alpha} \in \mathscr{G}$ by $\beta\left(H_{\alpha}\right)=2(\beta, \alpha) /(\alpha, \alpha)$ for $\beta \in \mathfrak{G}^{*}$. Let $W$ be the Weyl group of $\mathscr{G}$ in $g$. Denote by $M$ the Z-span of $W \theta$ (long roots).

Let $\mathbf{C}\left[t, t^{-1}\right]$ be the algebra of Laurent polynomials over $\mathbf{C}$ in an indeterminate $t$. We regard $\tilde{g}:=\mathbf{C}\left[t, t^{-1}\right] \otimes_{\mathbf{C}} g$ as an (infinite-dimensional) complex Lie algebra. Define the affine Lie algebra $\hat{g}$ as follows. Let $\hat{g}=\widetilde{g} \oplus \mathbf{C} c \oplus \mathbf{C d}$ and define the bracket by

$$
[x, y]_{\hat{g}}=[x, y]_{\tilde{g}}+\operatorname{Res}_{t=0}\left(\frac{d x}{d t}, y\right) c,[d, x]=t \frac{d x}{d t},[c, x]=0=[c, d],
$$

for $x, y \in \widetilde{g}$. The algebra $\hat{g}$ is an important example of a Kac-Moody algebra [5], [10]. Note that $\mathbf{C} c$ is the center of the algebra $\hat{g}$. The subalgebra $\hat{b}=$ $\mathfrak{G} \oplus \mathbf{C} c \oplus \mathbf{C} d$ is called the Cartan subalgebra of $\hat{g}$. For $\alpha \in \hat{b}^{*}$ set $\hat{g}_{\alpha}=$ $\{x \in \hat{g} \mid[h, x]=\alpha(h) x$ for $h \in \hat{b}\}$; then we have the root space decomposition $\hat{g}=\bigoplus \hat{g}_{\alpha}$.

Detine a nondegenerate symmetric bilinear form $($,$) on \hat{G}$ by $\left(h, h^{\prime}\right)$ is unchanged if $h, h^{\prime} \in \mathfrak{G} \subset \hat{\mathfrak{h}},(h, c)=(h, d)=0$ for $h \in \mathfrak{h},(c, c)=(d, d)=0$, $(c, d)=1$. We identify $\hat{b}$ with $\hat{b}^{*}$ by this form; then $b^{*}$ is identified with a subspace in $\hat{b}^{*}$ by $\alpha(c)=\alpha(d)=0$ for $\alpha \in \mathscr{G}^{*}$. For $\alpha \in \hat{\mathfrak{G}}^{*}$ set $\bar{\alpha}=\left.\alpha\right|_{\mathfrak{G}}$. so that $\bar{\alpha} \in \mathfrak{G}^{*} \subset \hat{\mathfrak{G}}^{*}$. Define $\delta \in \hat{\mathrm{f}}^{*}$ by $\delta(h)=0$ for $h \in \mathfrak{G}, \delta(c)=0, \delta(d)=1$.

Received by the editors April 7, 1980.

1980 Mathematics Subject Classification. Primary 17B65, 10 D12. 
The root system of $\hat{g}$ is defined to be the set

$$
\hat{\Delta}:=\left\{\alpha \in \hat{b}^{*} \mid \alpha \neq 0, \operatorname{dim} \hat{g}_{\alpha} \neq 0\right\} .
$$

The numbers mult $\alpha:=\operatorname{dim} \hat{g}_{\alpha}$ are as follows: mult $\alpha=1$ for $\alpha \in \Delta+\mathbf{Z} \delta,=l$ for $\alpha \in \mathbf{Z} \delta, \alpha \neq 0$, and $=0$ for all the other $\alpha \neq 0$. The set

$$
\hat{\Delta}_{+}=\{\alpha \in \hat{\Delta} \mid \alpha(d)>0\} \cup \Delta_{+}
$$

is the positive root system, and $\hat{\Pi}=\left\{\alpha_{0}:=\delta-\theta, \alpha_{1}, \ldots, \alpha_{l}\right\}$ is the set of simple roots. The set of dual simple roots is

$$
\left\{h_{0}:=c-H_{\theta}, h_{i}:=H_{\alpha_{i}}, i=1, \ldots, l\right\} .
$$

We define a reflection $r_{i} \in G L\left(\hat{G}^{*}\right)$ by $r_{i}(\lambda)=\lambda-\lambda\left(h_{i}\right) \alpha_{i}, i=0, \ldots, l$, $\lambda \in \hat{\mathfrak{G}}^{*}$. The Weyl group $\hat{W}$ of $\hat{g}$ is the subgroup in $G L\left(\hat{G}^{*}\right)$ generated by all $r_{i}, i=0, \ldots, l$ We regard $W$ as the subgroup of $\hat{W}$ generated by all $r_{i}$, $i=1, \ldots, l$. For $\alpha \in \mathfrak{G}^{*}$ set

$$
t_{\alpha}(\lambda)=\lambda+\lambda(c) \alpha-((\lambda, \alpha)+1 / 2 \lambda(c)(\alpha, \alpha)) \delta, \lambda \in \hat{b}^{*}
$$

and set $T=\left\{t_{\alpha} \mid \alpha \in M\right\}$. Then one has

$$
\hat{w}=W \times T .
$$

2. Set $\hat{P}=\left\{\lambda \in \hat{G}^{*} \mid \lambda(d)=0 ; \lambda\left(h_{i}\right) \in \mathbf{Z}, i=0, \ldots, l\right\}, \hat{P}_{+}=$ $\left\{\lambda \in \hat{P} \mid \lambda\left(h_{i}\right) \geqslant 0, i=0, \ldots, l\right\}, P=\hat{P} \cap \zeta^{*}$. Define $\rho \in \hat{G}^{*}$ by $\rho\left(h_{i}\right)=1$, $i=0, \ldots, l, \rho(d)=0$, and set $g=\rho(c)$.

Fix $\Lambda \in \hat{P}_{+}$; there exists a unique irreducible $\hat{g}$-module $L(\Lambda)$ (the highest weight module) which admits a nonzero $v \in L(\Lambda)$ such that $\hat{g}_{\alpha}(v)=0$ for $\alpha \in \hat{\Delta}_{+}$and $h(v)=\Lambda(h) v$ for $h \in \hat{\mathfrak{h}}$. For $\lambda \in \hat{\mathfrak{g}}^{*}$ set $L(\Lambda)_{\lambda}:=\{v \in L(\Lambda) \mid h(v)$ $=\lambda(h) v$ for $h \in \hat{b}\}$, mult $\Lambda_{\Lambda}(\lambda):=\operatorname{dim} L(\Lambda)_{\lambda}$. If mult $_{\Lambda}(\lambda) \neq 0, \lambda$ is called a weight of $L(\Lambda)$. We have $L(\Lambda)=\bigoplus L(\Lambda)_{\lambda}$, and mult $_{\Lambda}(w \lambda)=\operatorname{mult}_{\Lambda}(\lambda)$ for $w \in \hat{W}$ (see [7] for details). The number $m:=\Lambda(c)$ is called the level of the module $L(\Lambda)$; this is a nonnegative integer, which is 0 if and only if $\operatorname{dim} L(\Lambda)=1$.

With respect to $d$ we have the eigenspace decomposition

$$
L(\Lambda)=\bigoplus_{k \in Z_{+}} L^{(-k)},
$$

where $\operatorname{dim} L^{(-k)}<\infty$. Consider the domain $D:=\{z+\tau d+u c \mid z \in \mathfrak{G} ; \tau, u \in \mathbf{C}$, $\operatorname{Im} \tau>0\} \subset \hat{b}$. We define the character of the $\hat{g}$-module $L(\Lambda)$ by

$$
\operatorname{ch} L(\Lambda)(z, \tau, t):=\left.\sum_{k \geqslant 0} \operatorname{tr} \exp (-2 \pi i(z+\tau d+u c))\right|_{L}(-k) .
$$

This is an absolutely convergent series defining a $\hat{W}$-invariant holomorphic function on $D$. The character can be written in terms of classical theta-functions 
$\Theta_{\lambda, n}$. For $\lambda \in \mathbf{Q} \otimes_{\mathrm{Z}} M$, and a positive integer $n, \Theta_{\lambda, n}$ is the holomorphic function on $D$ defined by

$$
\Theta_{\lambda, n}(z, \tau, u)=e^{-2 \pi i n u} \sum_{\gamma \in M+n^{-1} \lambda} e^{\pi i n|\gamma|^{2} \tau} e^{-2 \pi i n \gamma(z)} .
$$

The character formula from [6] can be rewritten as follows:

$$
e^{-2 \pi i \Lambda_{\Lambda} \tau} \operatorname{ch} L(\Lambda)(z, \tau, u)=\sum_{w \in W}(\operatorname{det} w) \Theta_{w(\bar{\Lambda}+\bar{\rho}), m+g} / \sum_{w \in W}(\operatorname{det} w) \Theta_{w(\bar{\rho}), g},
$$

where $r_{\Lambda}=(2 m+2 g)^{-1}|\Lambda+\rho|^{2}-(2 g)^{-1}|\rho|^{2}$.

Consider $L(\Lambda)$ as an 5 -module; one has

$$
L(\Lambda)=\bigoplus_{\lambda \in \mathfrak{G}^{*}} L^{\lambda}, \text { where } L^{\lambda}:=\{v \in L(\Lambda) \mid h(v)=\lambda(h) v \text { for } h \in \mathscr{G}\} .
$$

Let $\lambda \in \mathfrak{g}^{*}$ be such that $L^{\lambda} \neq 0$. Choose the minimal $n_{0} \in \mathbf{Z}$ such that $\operatorname{mult}_{\Lambda}\left(\lambda+\Lambda-\bar{\Lambda}-n_{0} \delta\right) \neq 0$. Set $r_{\Lambda}(\lambda):=-n_{0}-(2 m+2 g)^{-1}|\bar{\Lambda}+\bar{\rho}|^{2}+$ $(2 m)^{-1}|\lambda|^{2}+(2 g)^{-1}|\bar{\rho}|^{2}$, and (cf. [8])

$$
c_{\lambda}^{\Lambda}(\tau):=e^{2 \pi i r_{\Lambda}(\lambda) \tau} \sum_{k \geqslant 0}\left(\operatorname{dim} L^{\lambda} \cap L^{(-k)}\right) e^{2 \pi i k \tau}
$$

The function $c_{\lambda}^{\Lambda}$ is holomorphic on the upper half-plane; it is called a string function. We set $c_{\lambda}^{\Lambda}=0$ if $L^{\lambda}=0$. Note that $c_{\lambda}^{\Lambda}=c_{w(\lambda)}^{\Lambda}$ for $w \in W \ltimes m M$; these string functions are called equivalent. It is easy to see that, due to $\hat{W}$. invariance, one has (cf. [8] and [9])

$$
e^{-2 \pi i \Lambda_{\Lambda} \tau} \operatorname{ch} L(\Lambda)(z, \tau, u)=\sum_{\lambda \in P / m M} c_{\lambda}^{\Lambda}(\tau) \Theta_{\lambda, m}(z, \tau, u)
$$

Comparing (1) and (2) gives an identity, which together with the transformation law for theta functions [1] gives the following:

THEOREM 1. Let $\Lambda \in \hat{P}_{+}$be such that $m=\Lambda(c)>0$, and $\lambda \in P$.

Let $N$ be the least positive integer such that $N|\mu|^{2} \in 2 Z$ for $\mu \in P$. Then

(a) $c_{\lambda}^{\Lambda}\left(-\tau^{-1}\right)=(-i \tau)^{-l / 2} \sum_{\substack{\Lambda^{\prime} \in \hat{P}_{+} \\ \Lambda^{\prime}(c)=m \\ \lambda^{\prime} \in P / m M}} b\left(\Lambda, \lambda ; \Lambda^{\prime}, \lambda^{\prime}\right) c_{\lambda^{\prime}}^{\Lambda^{\prime}}(\tau)$

where

$$
\begin{array}{r}
b\left(\Lambda, \lambda ; \Lambda^{\prime}, \lambda^{\prime}\right)=i^{\mid \Delta+}|P / M|^{-1} m^{-l / 2}(m+g)^{-l / 2} \exp \left(2 \pi i m^{-1}\left(\lambda, \lambda^{\prime}\right)\right) \\
\sum_{w \in W}(\operatorname{det} w) \exp \left(-2 \pi i(m+g)^{-1}\left(\bar{\Lambda}+\bar{\rho}, w\left(\bar{\Lambda}^{\prime}+\bar{\rho}\right)\right)\right) .
\end{array}
$$


(b) $\eta(\tau)^{\operatorname{dim} g} c_{\lambda}^{\Lambda}(\tau)$ is a cusp-form of weight $\left|\Delta_{+}\right|$for $\Gamma(N m) \cap \Gamma(N(m+g))$, with multiplier system given in [12].

3. Theorem 1 allows one to compute the string functions in many interesting cases. For $0 \leqslant i \leqslant l$ define $\Lambda_{i} \in \hat{P}_{+}$by $\Lambda_{i}\left(h_{j}\right)=\delta_{i j}, \Lambda_{i}(d)=0$. We write $c_{n}^{m}(\tau)$ for $c_{\lambda}^{\Lambda}$, where $\Lambda=\Lambda_{m}, \lambda=\overline{\Lambda_{n}}$.

EXAMPLE 1. Suppose all roots of $g$ have equal length. Then all $\Lambda \in \hat{P}_{+}$ with $\Lambda(c)=1$ are conjugate under automorphisms and for each such $\Lambda$ there is, up to equivalence, a unique string function. Thus all nonzero string functions of the modules of level 1 are equal to $c_{0}^{0}(\tau)$. Using Theorem 1 , we obtain $c_{0}^{0}(\tau)=$ $\eta(\tau)^{-l}$, which is proved in [7] by a more complicated method. For the $A_{1}$ module $L\left(\Lambda_{0}\right)$ the weight multiplicities have been previously computed in [2].

EXAMPLE 2. Let $g$ be of type $B_{l}$ with roots labelled as in the diagram

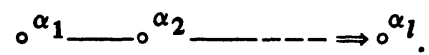

Then there are three distinct string functions for $\Lambda(c)=1$, and

$$
\begin{aligned}
& c_{l}^{l}(\tau)=\frac{\eta(2 \tau)}{\eta(\tau)^{l+1}}, \quad c_{0}^{0}(\tau)-c_{1}^{0}(\tau)=\frac{\eta(\tau / 2)}{\eta(\tau)^{l+1}}, \\
& c_{0}^{0}(\tau)+c_{1}^{0}(\tau)=\frac{1}{\eta(\tau / 2) \eta(\tau)^{l-2} \eta(2 \tau)}
\end{aligned}
$$

Similar results may be obtained for $g$ of types $G_{2}, F_{4}, C_{3}$, but the general case of $C_{l}$ seems difficult.

EXAMPLE 3. Theorem 1 can be generalized to the case of the Lie algebras from Tables 2 and 3 (in notations of [7]). For $\Lambda=\Lambda_{0}$ there is, up to equivalence, a unique string function, namely $c_{0}^{0}(\tau)$, given as follows: $\eta(\tau)^{-l}$ for $A_{2 l}^{(2)}$, $\eta(\tau)^{-l+1} \eta(2 \tau)^{-1}$ for $A_{2 l-1}^{(2)}, \eta(\tau)^{-1} \eta(2 \tau)^{-l+1}$ for $D_{l+1}^{(2)}, \eta(\tau)^{-2} \eta(2 \tau)^{-2}$ for $E_{6}^{(2)}$ and $\eta(\tau)^{-1} \eta(3 \tau)^{-1}$ for $D_{4}^{(3)}$.

4. The (generalized) Kostant partition function $K$ on $\hat{b}^{*}$ is defined by setting $K(\lambda)$ equal to the number of partitions of $\lambda$ as a sum of positive roots (from $\hat{\Delta}_{+}$), each root being taken with its multiplicity. The function $K$ may be used to compute mult ${ }_{\Lambda}(\lambda)[6]$, and hence to compute $c_{\lambda}^{\Lambda}(\tau)$.

We do this straightforward computation for $\hat{\mathrm{g}}=\hat{A}_{1}$. Then $\delta=\alpha_{0}+\alpha_{1}$, $\hat{\Delta}_{+}=\left\{(n+1) \delta, n \delta+\alpha_{0}, n \delta+\alpha_{1}\right.$, where $\left.n \geqslant 0\right\}$, and mult $\alpha=1$ for all $\alpha \in \hat{\Delta}_{+}$. We have the formula

$$
K\left(n_{0} \alpha_{0}+n_{1} \alpha_{1}\right)=\sum_{k \geqslant 0}(-1)^{k} p^{(3)}\left((k+1) n_{0}-k n_{1}-\frac{1}{2} k(k+1)\right)
$$

where $p^{(3)}(n)$ is defined by $\Sigma_{n} p^{(3)}(n) X^{n}=\Pi_{n>1}\left(1-X^{n}\right)^{-3}$. Similar formulas exist for all $\hat{\mathrm{g}}$, but are tractable only for type $\hat{A}_{l}$. They are obtained using the results from [11]. Applying (3) we obtain 
THEOREM 2. Let $L(\Lambda)$ be an $\hat{A}_{1}$-module of level $m>0$, and let $\lambda \in P$, $c_{\lambda}^{\Lambda} \neq 0$. Set $a(\lambda)={ }^{t}\left(\left(\Lambda\left(h_{1}\right)+1\right)(2 m+2)^{-1}, \lambda\left(h_{1}\right)(2 m)^{-1}\right) \in \mathbf{R}^{2}$. Let $F$ be the quadratic form $F(x, y)=(m+2) x^{2}-m y^{2}$ in $\mathbf{R}^{2}$. For $v \in \mathbf{R}^{2}$ set $\operatorname{sign} v=$ $\operatorname{sign}(\sqrt{m+2} x+\sqrt{m y})$. Let $G_{0}$ be the subgroup of $\operatorname{SL}(2, \mathbf{R})$ generated by

$$
\left(\begin{array}{ll}
m+1 & m \\
m+2 & m+1
\end{array}\right)^{2}
$$

Then

$$
\eta(\tau)^{3} c_{\lambda}^{\Lambda}(\tau)=\sum_{\substack{v \in \mathrm{z}^{2}+a(\lambda) \\ F(v)>0 \\ v \bmod G_{0}}}(\operatorname{sign} v) e^{2 \pi i \tau F(v)} .
$$

The function (5) is a modular form of a type studied by Hecke [4]. Comparing (5) with Example 1 for $l=1$ we obtain an identity for $\eta(\tau)^{2}$, which appears in [4]. Comparing (5) with Example (b) from [7, p. 132] (see also [2, Corollary 5.2]) we obtain a new identity

$$
\eta(\tau) \eta(2 \tau)=\sum_{\substack{m, n \in Z \\ n \geq 3|m|}}(-1)^{n} \exp 2 \pi i \tau\left[\frac{(2 n+1)^{2}}{8}-4 m^{2}\right] .
$$

\section{REFERENCES}

1. M. Eichler, Introduction to the theory of algebraic numbers and functions, Academic Press, New York, 1966.

2. A. Feingold and J. Lepowsky, The Weyl-Kac character formula and power series identities, Adv. in Math. 29 (1978), 271-309.

3. I. B. Frenkel and V. G. Kac, Basic representations of affine Lie algebras and dual resonance models, Invent. Math. (to appear).

4. E. Hecke, Über einen Zusammenhang zwischen elliptischen Modulfunktionen und indefiniten quadratischen Formen, Mathematische Werke, Vandenhoeck and Ruprecht, Göttingen, 1959 , pp. 418-427.

5. V. G. Kac, Simple irreducible graded Lie algebras of finite growth, Math. USSRIzvestija 2 (1968), $1271-1311$.

6. - Infinite-dimensional Lie algebras and Dedekind's $\eta$-function, J. Functional Anal. Appl. 8 (1974), 68-70.

7. - Infinite-dimensional algebras, Dedekind's $\eta$-function, classical Möbius function and the very strange formula, Adv. in Math. 30 (1978), 85-136.

8. - An elucidation of "Infinite-dimensional algebras and the very strange formula". $E_{8}^{(1)}$ and the cube root of the modular invariant $j$, Adv. in Math. 35 (1980), 264-273.

9. E. Looijenga, Root systems and elliptic curves, Invent. Math. 38 (1976), 17-32.

10. R. V. Moody, A new class of Lie algebras, J. Algebra 10 (1968), 211-230.

11. D. H. Peterson, Kostant-type partition functions (to appear).

12. V. G. Kac and D. H. Peterson (manuscript in preparation).

DEPARTMENT OF MATHEMATICS, MASSACHUSETTS INSTITUTE OF TECHNOLOGY, CAMBRIDGE, MASSACHUSETTS 02139 